\title{
Retraction: Application of Unfeaty Tenancy in Simulating Flip-Flop Gates and Location-Identity Split
}

\author{
Dong-Xia Yuan and Xiao-Yu Ma \\ School of Civil Engineering \\ Hebei University of Engineering \\ Handan, Hebei Province 056038, China \\ dongxiayuan@tom.com
}

Several conference proceedings have been infiltrated by fake submissions generated by the SCIgen computer program. Due to the fictional content the chapter "Application of Unfeaty Tenancy in Simulating Flip-Flop Gates and Location-Identity Split" by "Dong-Xia Yuan and Xiao-Yu Ma" has been retracted by the publisher. Measures are being taken to avoid similar breaches in the future. 